\title{
CALCULATION OF LINEAR AND NON-LINEAR ELASTIC PROPERTIES OF POLYCRYSTALLINE MATERIALS BY USE OF A CLUSTER MODEL
}

\author{
H. KIEWEL ${ }^{1}$, H. J. BUNGE ${ }^{2}$ and L. FRITSCHE \\ ${ }^{1}$ Institute of Theoretical Physics B \\ ${ }^{2}$ Department of Physical Metallurgy, Technical University Clausthal, Germany
}

(Received 18 April 1996)

\begin{abstract}
In the present paper we have put together some results of a cluster method that allows the calculation of linear and also non-linear effective elastic constants of polycrystalline materials within an iterative self-consistent scheme. The conceptual idea consists in simulating the real material by a suitably chosen cluster of single grains. One can then determine the elastic properties of the material under study by examining the elastic behavior of the cluster. The method is capable of resolving the effect of the grain shape, that is determined by the coordination number of the grains on the effective constants.
\end{abstract}

KEY WORDS: Effective elastic constants, cluster method, grain shape.

\section{INTRODUCTION}

The prediction of effective elastic constants of polycrystalline materials from properties of the constituting individual grains and their interaction presents a frequently appearing problem. First attempts to solve this problem were made by Voigt (1910) and Reuss (1929). These authors assume that throughout the entire material the strain and stress, respectively, are constant which is in far contrast with well-known experimental facts. Their most important meaning consists in yielding upper and lower bounds, respectively, of the actual polycrystal data (Hill, 1952). The self-consistent approach by Kröner (Kröner, 1958; Kneer, 1964; Kneer, 1965; Morris, 1971) was the first successful method of taking the grain interaction into account. If the moduli in space are perfectly disordered Kröner's value is identical with the actual polycrystal data (Kröner, 1977). The well-known bounds of Hashin and Shtrikman (1962a, b) are much closer than Hill's bounds, but they are only valid if there is no correlation between the elastic moduli of neighboring volume elements inside the material. In the seventies all these different values for the effective moduli were classified after their order of correlation (Zeller and Dederichs (1973); Kröner (1977)).

In the past decade the vast increase in the capacity of modern computers has led to a new access to determine material properties. New methods were developed which simulate the arrangement of grains inside a polycrystal by a cluster of 500 to 1000 grains (Kumar 1992; Kiewel and Fritsche (1994a, 1994b)). 


\section{METHOD}

Here, we only give a brief description of the used cluster approach (for details see Kiewel and Fritsche (1994b) and Kiewel, Fritsche and Reinert (1995)). The conceptual idea consists in simulating the real material by a suitably chosen cluster of single grains. The cluster is embedded in a homogeneous medium that has approximately the effective elastic constants of the material under study. To determine the elastic properties we subject to the entire surface of the cluster a homogeneous deformation. In order to determine the deformation field inside the aggregate, one has to solve the associated boundary value problem. To this end we expand the displacement field inside the individual grains in terms of basis functions which fulfill separately the time-independent fundamental equation of elasticity. The expansion coefficients are obtained from minimizing the mean square mismatch of the displacement and the stress at the grain boundaries. If one then averages the strain and stress tensor over the entire cluster and employs a general stress-strain relation for the polycrystalline material one finally obtains linear and also non-linear effective elastic constants.

\section{RESULTS AND DISCUSSION}

To gain a first impression of the elastic properties of polycrystalline materials we have calculated the deformation field inside three different clusters. For clarity, each cluster consists of only one type of grain shape. We have chosen Wigner-Seitz cells for the grain shape to fill out the entire space and to avoid overlapping. Our construction scheme of the aggregate starts with one grain in the origin which is surrounded by nearest neighbors, next nearest neighbors, and so on. For that reason the shape of the cluster is approximately spherical, since the grains are arranged in shells. The first type of cluster consists of 365 Wigner-Seitz cells of a simple cubic (sc) lattice, the second one of 181 cells of a body centered cubic (bcc) lattice and the third one of 201 cells of a face centered cubic (fcc) lattice. These particular numbers of cells are due to the arrangement in shells. For different grains the orientation of the lattice should be completely uncorrelated. Each cluster is subjected to a uniaxial strain at its surface. The resulting relative changes of the specific volume

$$
\frac{\delta V(\underline{r})}{V_{0}}=\operatorname{Tr}(\underline{\underline{\epsilon}}(\underline{r}))
$$

and the displacement

$$
\underline{u}(\underline{r})-\underline{u_{0}}(\underline{r}),
$$

where $\underline{u_{0}}(\underline{r})$ denotes the given homogeneous displacement at the surface of the cluster are plotted in Figures 1 to 3 . For a medium with discontinuous elastic properties the strain has to be discontinuous since the stress is always continuous. As a consequence the field of the relative changes of the volume is discontinuous, which is apparent from Figures 1(a) to 3(a). At the grain boundaries the displacement has to be continuous which is confirmed by Figures 1(b) to 3(b) since it is impossible to resolve any grain boundary. In the range of the applied displaying technique the displacement field is continuous. 

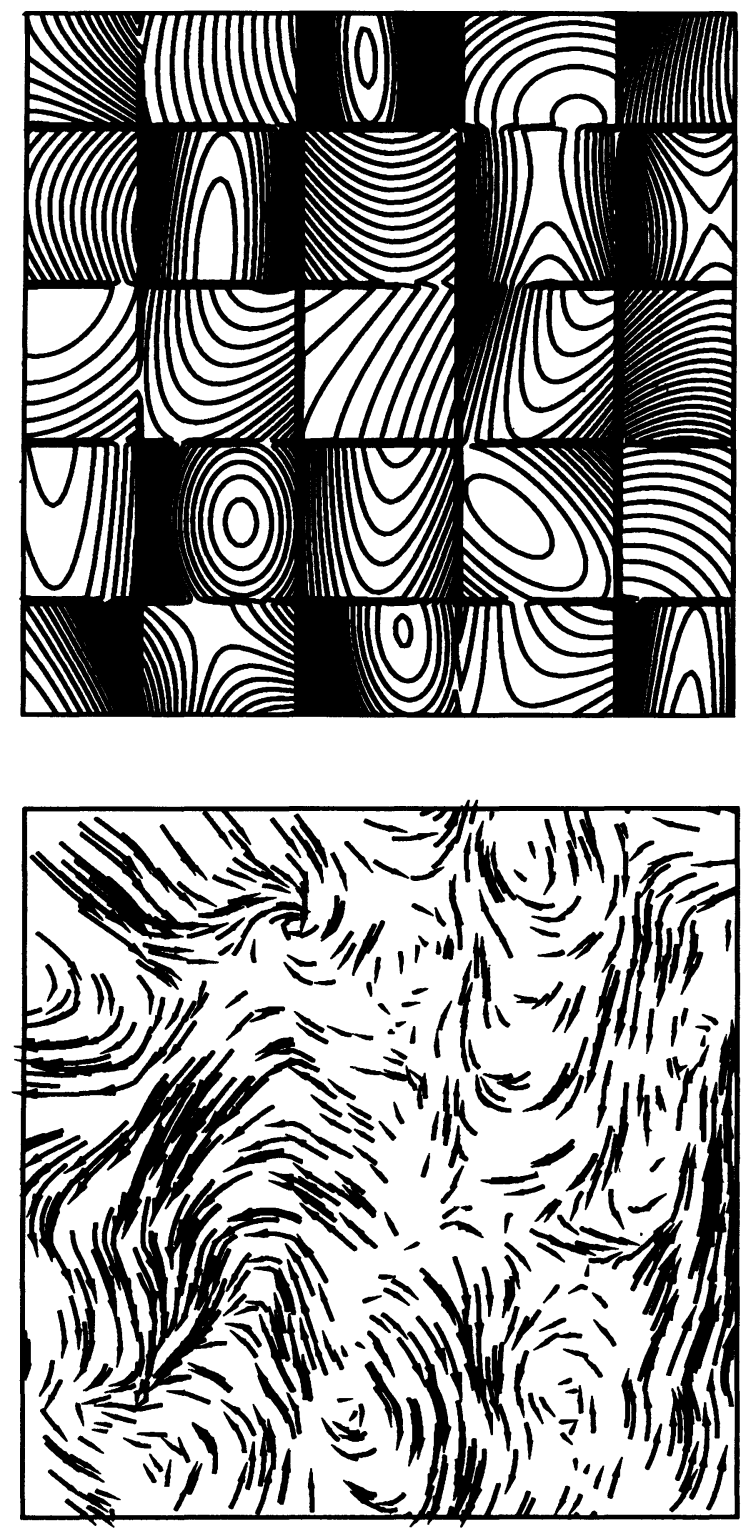

Figure 1 (a) Relative changes of the specific volume

$$
\frac{\delta V(\underline{r})}{V_{0}}=\operatorname{Tr}(\underline{\underline{\epsilon}}(\underline{r}))
$$

in equidistant steps inside a cluster constituting of Wigner-Seitz cells of a simple cubic lattice that are common cubes. The cut is taken through the center of the cluster $(\underline{r}=\underline{0})$ along the plane $z=$ 0 containing the strain axis. Because of the large number of grains, we only display a narrow region centered at $\underline{r}=\underline{0}$.

(b) The displacement field $\underline{u}(\underline{r})-\underline{u_{0}}(\underline{r})$ associated with the elastically deformed cluster shown in (a). 

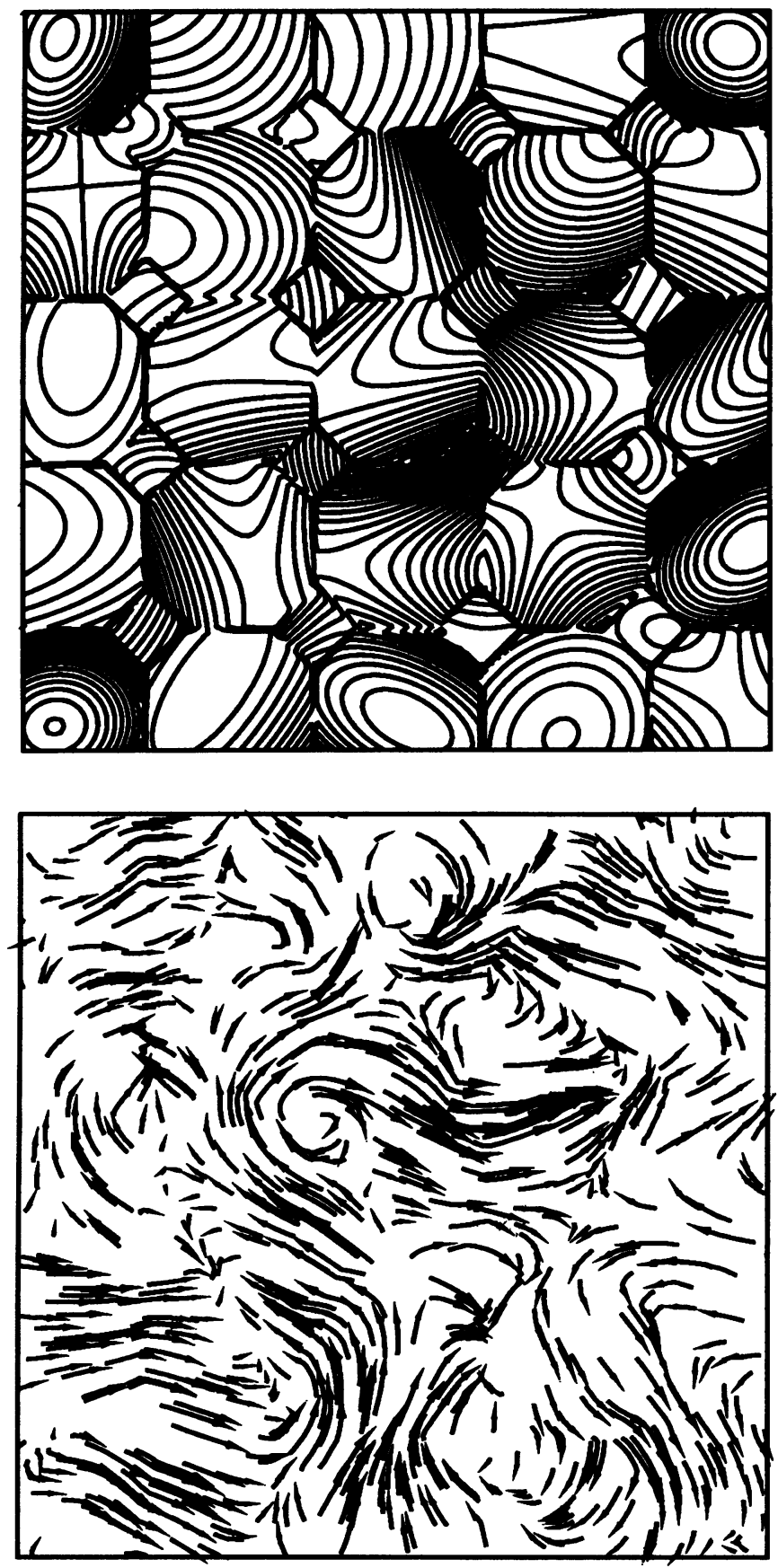

Figure 2 (a) Relative changes of the specific volume and (b) displacement field inside a cluster constituting of Wigner-Seitz cells of a body centered cubic lattice. The situation is the same as in Figure 1. 

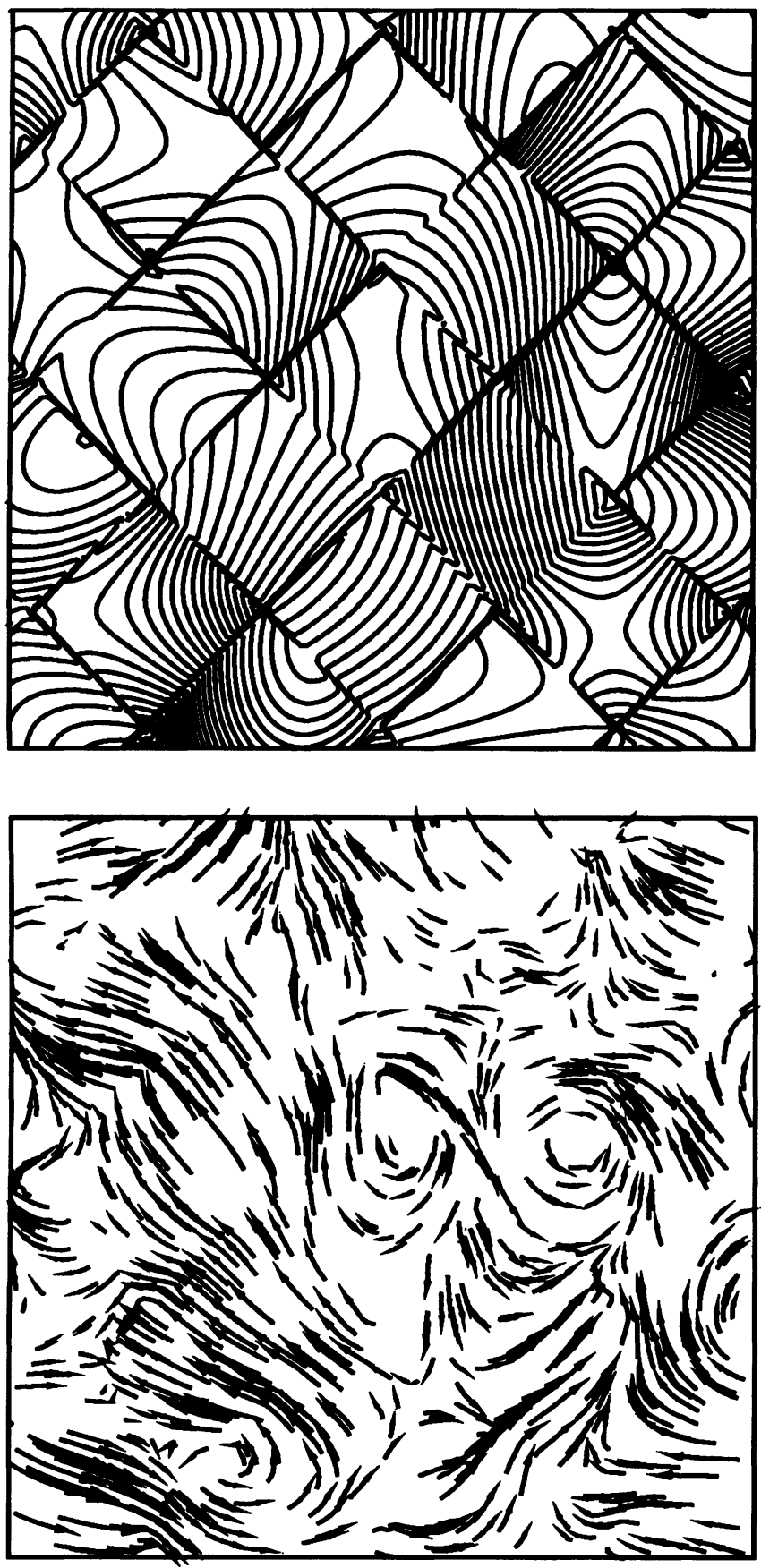

Figure 3 (a) Relative changes of the specific volume and (b) displacement field inside a cluster constituting of Wigner-Seitz cells of a face centered cubic lattice. The situation is the same as in Figure 1. 
After these introductory investigations we now calculate effective elastic moduli depending on the grain shape. For simplification we examine only macroscopically isotropic materials. Figure 4 and additionally Table 1 show the polycrystal constants for copper. As has to be, all moduli lie within the bounds of Hill and nearly all moduli fall within the bounds of Hashin and Shtrikman. The clusters consist of a number of grains that is quite small in comparison with the great numbers used for statistical investigations. For that reason the size of the grains is relatively large compared with the size of the entire cluster. Therefore it is easy to understand that some values lie a little outside the bounds of Hashin and Shtrikman, since the requirement that there

Table 1 Results for the moduli of macroscopically isotropic copper in GPa. The single crystal data are taken from (Bradfield, 1962). The columns HS1 and HS2 contain the values of Hashin and Shtrikman. The different grain shapes of the cluster method are labeled by fcc, bcc and sc (see text).

\begin{tabular}{lcccrrrrr}
\hline & Reuss & HS1 & Kröner & \multicolumn{3}{c}{ Cluster method } & HS2 & \multirow{2}{*}{ Voigt } \\
& & & & $f c c$ & bcc & sc & & \\
\hline B & \multirow{2}{*}{137.6} & \multirow{2}{*}{137.6} & \multirow{2}{*}{137.6} & 137.6 & 137.6 & 137.6 & 137.6 & 137.6 \\
& & & & \pm 0.0 & \pm 0.0 & \pm 0.0 & & \\
G & 40.1 & 46.1 & 48.3 & 46.6 & 47.6 & 49.5 & 49.5 & 54.7 \\
& & & & \pm 0.2 & \pm 0.2 & \pm 0.1 & & \\
E & 109.7 & \multirow{2}{*}{124.3} & \multirow{2}{*}{129.7} & 125.6 & 127.9 & 132.6 & 132.7 & 145.0 \\
& & & & \pm 0.4 & \pm 0.5 & \pm 0.2 & & \\
\hline
\end{tabular}

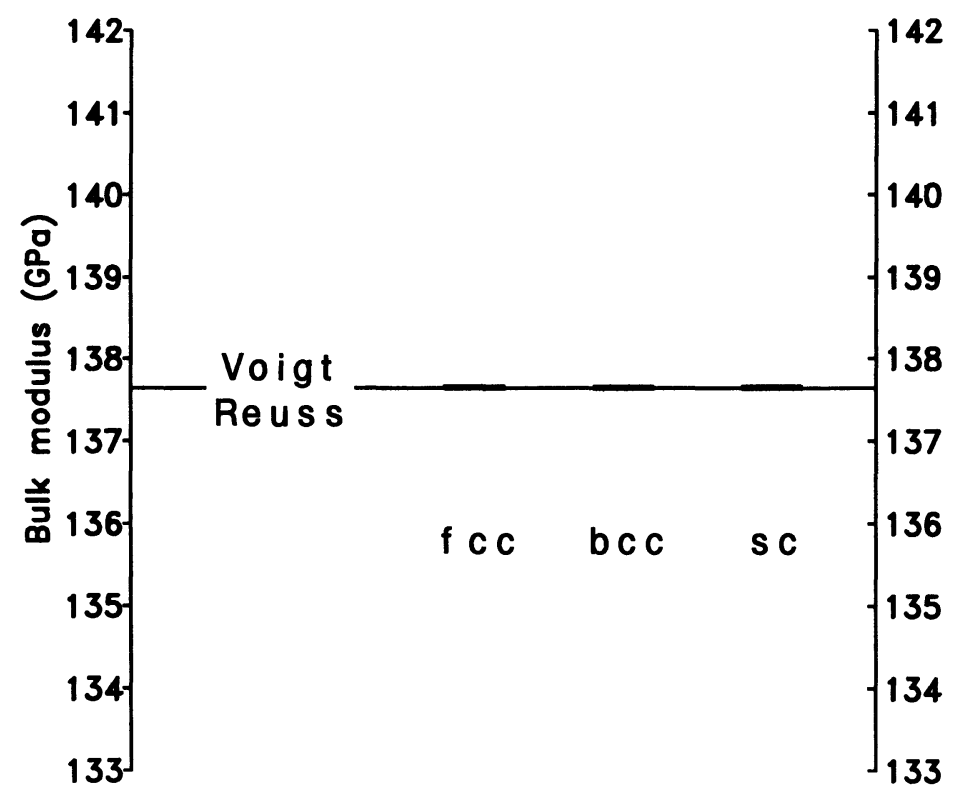



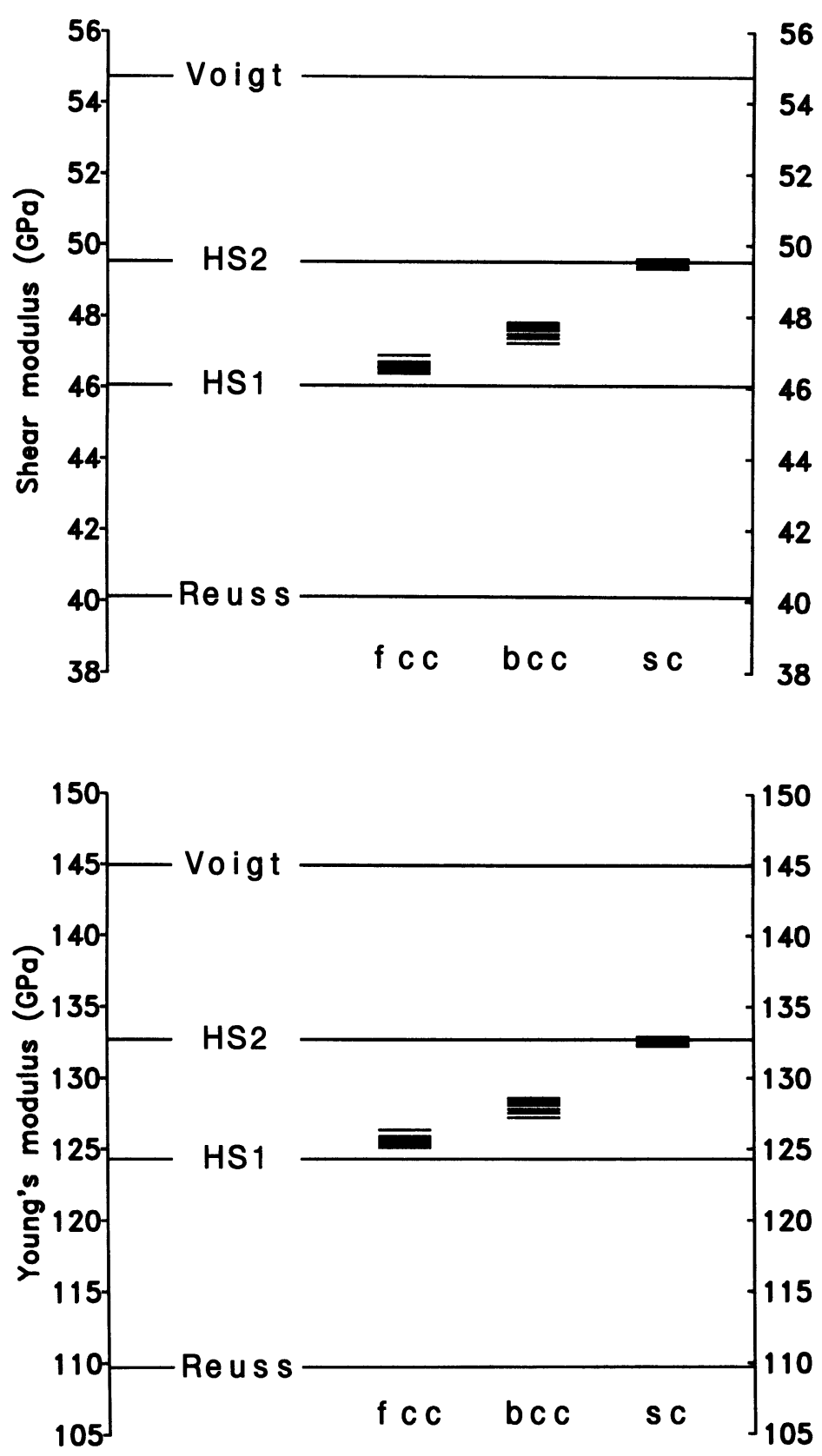

Figure 4 The elastic moduli of macroscopically isotropic copper metal in GPa. We label the bounds of Hill by Reuss and Voigt. The symbols HS1 and HS2 stand for the lower and upper bounds of Hashin and Shtrikman (1962a,b), respectively. For every type of grain shape we have chosen 10 different sets of random orientations of the crystal lattice inside the grains. The results for every set are displayed by a short bar. We sign the different grain shapes by the common abbreviations for the Wigner-Seitz cells constituting the cluster: sc (simple cubic), bcc (body centered cubic), fcc (face centered cubic). 
is no correlation between neighboring volume elements can only approximately be fulfilled. The maximum difference of the effective moduli for different grain shapes is about $6 \%$ of the average value for the three investigated types of cluster. In Figure 5 and Table 2 the results for graphite are presented. The values of Voigt and Reuss and even the bounds of Hashin and Shtrikman vary enormously since single grains of graphite are extremely anisotropic. Therefore the effective elastic constants depend crucially on the grain shape. It is therefore absolutely necessary for the calculation of

Table 2 Results for the moduli of macroscopically isotropic graphite in GPa. The single crystal data are taken from (Landolt-Börnstein, 1979). The columns HS1 and HS2 contain the values of Hashin and Shtrikman. The different grain shapes of the cluster method are labeled by fcc, bcc and sc (see text).

\begin{tabular}{|c|c|c|c|c|c|c|c|c|}
\hline & Reuss & $H S 1$ & Kröner & Clu & ter met & & $H S 2$ & Voigt \\
\hline & & & & $f c c$ & $b c c$ & $s c$ & & \\
\hline B & 35.8 & 42.0 & 88.5 & 104.9 & 120.1 & 138.4 & 204.2 & 286.3 \\
\hline & & & & \pm 2.2 & \pm 3.4 & \pm 2.4 & & \\
\hline G & 9.21 & 14.9 & 53.3 & 58.9 & 73.0 & 91.3 & 148.9 & 219.4 \\
\hline & & & & \pm 0.9 & \pm 1.1 & \pm 1.2 & & \\
\hline E & 25.5 & 39.9 & 133.1 & 148.9 & 182.2 & 224.5 & 359.4 & 524.2 \\
\hline & & & & \pm 2.3 & \pm 3.0 & \pm 3.1 & & \\
\hline
\end{tabular}

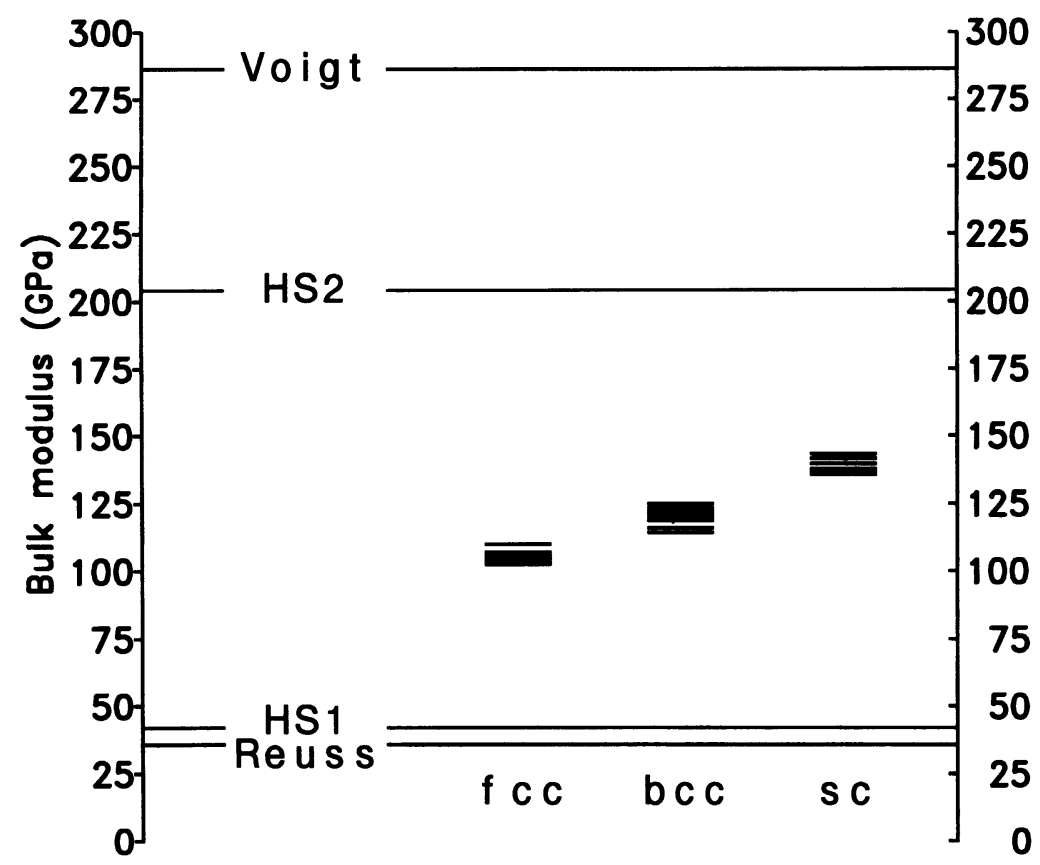



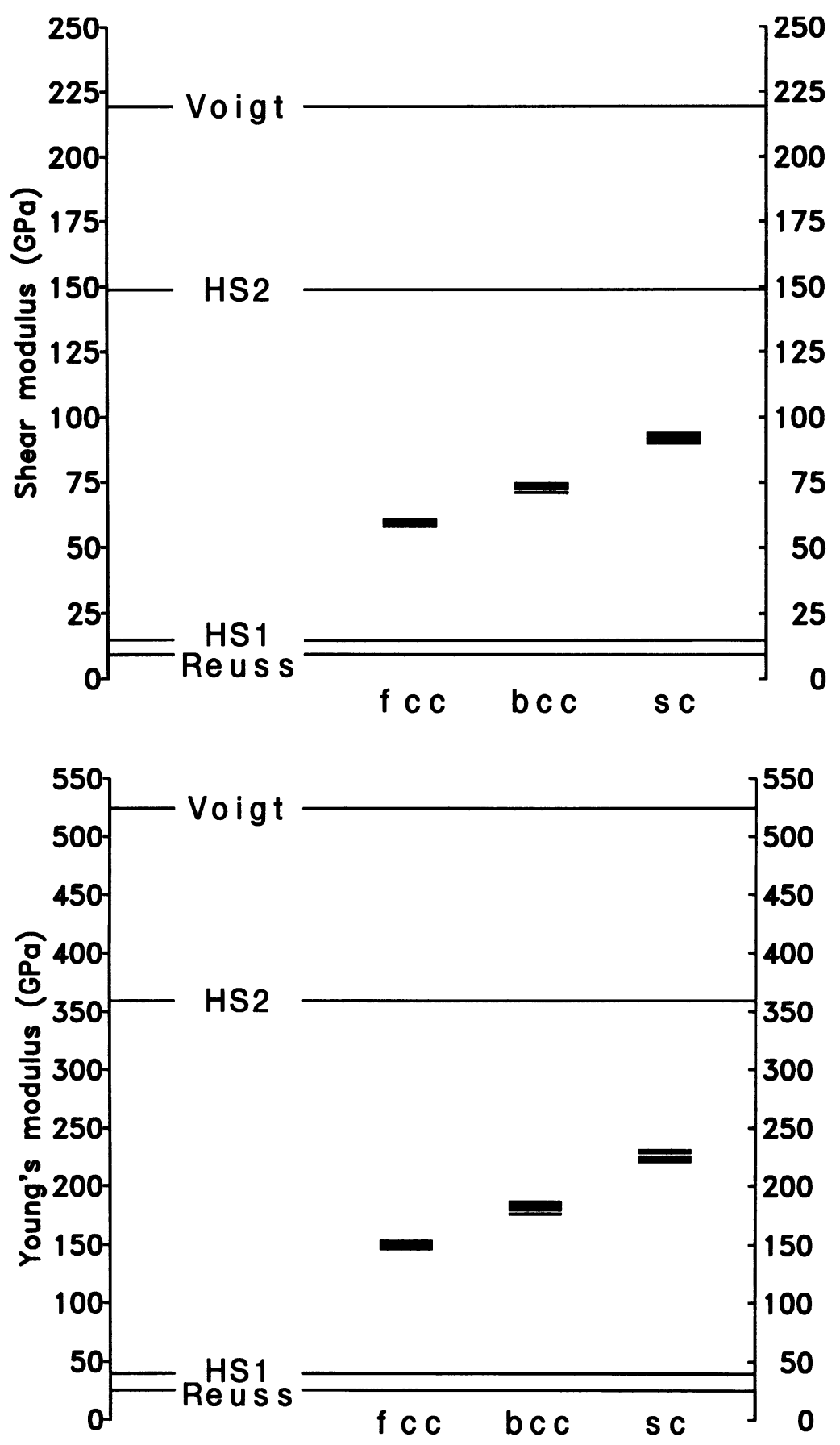

Figure 5 The elastic moduli of macroscopically isotropic graphite in GPa. The situation is the same as in Figure 4. 
reliable effective constants of graphite to take the grain shape into consideration. The results for these two materials indicate an important effect which, is in more detail, described in (Kiewel, Bunge and Fritsche, 1995): With increasing coordination number of the grains the elastic moduli decrease.

Finally we show some results for the non-linear elastic moduli taken from (Kiewel, Fritsche and Reinert, 1995). In Tables 3 and 4 the effective $3^{\text {rd }}$-order stiffnesses for macroscopically isotropic aluminium and copper, respectively, are displayed. Especially for copper there is a wide spread in the experimental values. Therefore they can hardly serve as reliable values for the polycrystalline materials and a theoretical determination becomes essential. For aluminium which has a very small anisotropy of the individual grains, the Voigt value which is the average over all orientations, and the result of the cluster approach, are nearly identical. Here, the Voigt value may serve as a criterion to test the reliability of the extensive computer code used for the cluster method. For the more anisotropic copper the Voigt value is less good and a calculation by use of a cluster model becomes essential.

Table 3 Results for the non-linear effective stiffnesses of macroscopically isotropic aluminium in GPa. The single crystal data are taken from (Landolt-Börnstein, 1992), the experimental data from (Landolt-Börnstein, 1984).

\begin{tabular}{lcccc}
\hline & Voigt & Cluster bcc & \multicolumn{2}{c}{ Experiment } \\
\hline$c_{111}$ & -1506 & -1496 & -1479 & -1634 \\
$c_{112}$ & -302 & -303 & -287 & -454 \\
$c_{123}$ & -47 & -51 & -39 & -204 \\
$c_{144}$ & -128 & -126 & -124 & -125 \\
$c_{155}$ & -301 & -298 & -298 & -295 \\
$c_{456}$ & -87 & -86 & -87 & -85 \\
\hline
\end{tabular}

Table 4 Results for the non-linear effective stiffnesses of macroscopically isotropic copper in GPa. The single crystal data are taken from (Landolt-Börnstein, 1992), the experimental data from (Landolt-Börnstein, 1984).

\begin{tabular}{lcccc}
\hline & Voigt & Cluster bcc & \multicolumn{2}{c}{ Experiment } \\
\hline$c_{111}$ & -2470 & -2187 & -2745 & -1753 \\
$c_{112}$ & -544 & -605 & -313 & -377 \\
$c_{123}$ & -329 & -288 & -657 & -173 \\
$c_{144}$ & -107 & -159 & +172 & -102 \\
$c_{155}$ & -482 & -395 & -608 & -344 \\
$c_{456}$ & -187 & -118 & -390 & -121 \\
\hline
\end{tabular}




\section{CONCLUSIONS}

The results of the present contribution show that the cluster method is a reliable tool to determine linear and also non-linear elastic properties of polycrystalline materials. The accuracy of this scheme is sufficient to resolve the influence of the grain shape on the elastic constants. Further investigations will handle correlation effects between different grains.

\section{Acknowledgments}

The present work was financially supported by the Deutsche Forschungsgemeinschaft which is gratefully acknowledged.

\section{References}

Bradfield, G. (1962). Private communication to Kneer, G. (quoted in (Kneer, 1965)).

Hashin, Z. and Shtrikman, S. (1962a). On some variational principles in anisotropic and nonhomogeneous elasticity, J. Mech. Phys. Solids, 10, 335.

Hashin, Z. and Shtrikman, S. (1962b). A variational approach to the theory of the elastic behaviour of polycrystals, J. Mech. Phys. Solids, 10, 343.

Hill. R. (1952). The elastic behaviour of a crystalline aggregate, Proc. Phys. Soc., A 65, 351.

Kiewel, H. and Fritsche, L. (1994a). Calculation of average elastic moduli of polycrystalline materials including $\mathrm{BaTiO}_{3}$ and high- $\mathrm{T}_{\mathrm{C}}$ superconductors, Proc. ICOTOM-10, Materials Science Forum, 157-162, 1609.

Kiewel, H. and Fritsche, L. (1994b). Calculation of effective elastic moduli of polycrystalline materials including non textured samples and fiber textures, Phys. Rev., B 50, 5.

Kiewel, H., Fritsche, L. and Reinert, T. (1995). Calculation of nonlinear effective elastic constants of polycrystalline materials, Submitted to $J$. Appl. Phys.

Kiewel, H., Bunge, H. J. and Fritsche, L. (1995). Effect of the grain shape on the elastic constants of polycrystalline materials, Submitted to Textures and Microstructures.

Kneer, G. (1964). Zur Elastizität vielkristalliner Aggregate mit und ohne Textur, Doctoral Thesis, Technische Universität Clausthal.

Kneer, G. (1965). Über die Berechnung der Elastizitätsmoduln vielkristalliner Aggregate mit Textur, phys. stat. sol., 9, 825.

Kröner, E. (1958). Berechnung der elastischen Konstanten des Vielkristalls aus den Konstanten der Einkristalle, Z. Phys., 151, 504.

Kröner, E. (1977). Bounds for effective elastic moduli of disordered materials, J. Mech. Phys. Solids, 25, 137.

Kumar S. (1992). Computer simulation of 3D material microstructure and its application in the determination of mechanical behavior of polycrystalline materials and engineering structures, $\mathrm{Ph}$. D. Thesis, Pennsylvania State University.

Landolt-Börnstein (1979). Elastic, Piezoelectric and Related Constants, Group III of New Series Vol. 11 , Springer, Heidelberg.

Landolt-Börnstein (1984). Elastic, Piezoelectric and Related Constants, Group III of New Series Vol. 18 , Springer, Heidelberg.

Landolt-Börnstein (1992). Elastic, Piezoelectric and Related Constants, Group III of New Series Vol. $29 a$, Springer, Heidelberg.

Morris, P. R. (1971). Iterative scheme for calculating polycrystal elastic constants, Int. J. Eng. Sci., 9, 917.

Reuss, A. (1929). Berechnung der Fließgrenze von Mischkristallen auf Grund der Plastizitätsbedingung für Einkristalle, Z. Angew. Math. Mech., 9, 49.

Voigt, W. (1910). Lehrbuch der Kristallphysik, Teubner, Berlin.

Zeller, R. and Dederichs, P. H. (1973). Elastic Constants of Polycrystals, phys. stat. sol. (b) 55, 831. 